\title{
Données probantes à l'appui de la création de services de pharmacie : dans quelle mesure en avons-nous besoin?
}

\author{
par Scot H Simpson
}

$\mathrm{N}$ ous avons tous à composer avec des ressources limitées en temps, en personnel et en financement dans nos divers milieux de pratique. Malgré cela, les demandes et les attentes sont nombreuses en termes de création et d'expansion des services de pharmacie. Ces demandes viennent de diverses sources, dont les normes d'agrément, notamment les Pratiques organisationnelles requises d'Agrément Canada ${ }^{1}$, les collègues de travail, nos propres intérêts en matière de pratique clinique et d'ailleurs. Les bases de nouveaux services de pharmacie ou de services étendus devraient idéalement reposer sur des données irréfutables issues d'études cliniques aléatoires ayant démontré des bienfaits en termes de résultats cliniques et d'avantages économiques solides prouvant que le maximum de bienfaits est obtenu à partir des ressources utilisées². Cependant, nous n'avons bien souvent pas le luxe d'avoir de tels niveaux de données probantes, ce qui soulève la question à savoir : Combien de données probantes faut-il pour justifier la création d'un nouveau service de pharmacie?

Il existe deux pôles complètement opposés sur la nécessité d'avoir des données probantes robustes issues d'études cliniques pour nous aider à guider nos décisions. Le premier est que nous ne devrions mettre en place que des programmes étayés par des données probantes issues d'études cliniques aléatoires ayant démontré des améliorations significatives en matière de résultats cliniques. Cette approche me rappelle un examen systématique de Smith et Pell ${ }^{3}$. Bien que ces auteurs voulaient vérifier si les parachutes étaient efficaces pour prévenir des événements indésirables majeurs liés aux «menaces gravitationnelles » en résumant les données probantes d'études comparatives aléatoires, une recherche exhaustive de la littérature n'a pas permis de trouver de telles études. Même en l'absence de ce niveau de données probantes par excellence, beaucoup d'entre nous ont déjà sauté d'un avion ou d'une autre structure et étaient sûrs que le parachute réduirait significativement le risque d'événement indésirable.
Le second pôle, diamétralement opposé au premier, est d'adopter de bonnes idées plus ou moins étayées de données probantes, mais qui sont "pleines de gros bon sens ». Dans mon cours de pharmacoépidémiologie, j'utilise des exemples des études CAST (Cardiac Arrhythmia Suppression Trial) ${ }^{4}$, HOPE (Heart Outcomes Prevention Evaluation) ${ }^{5}$ et DREAM (Diabetes Reduction Assessment with Ramipril and Rosiglitazone Medication) ${ }^{6}$ pour illustrer le besoin de soins dans notre quête d'une idée qui a du sens. Dans ces deux exemples, la suppression de rythmes ventriculaires anormaux secondaires à un infarctus du myocarde (étude CAST) et l'emploi d'inhibiteurs de l'enzyme de conversion de l'angiotensine pour prévenir le diabète (études HOPE et DREAM), les cliniciens étaient disposés à adopter des théories pharmacologiques jusqu'à ce que des études cliniques aléatoires aient démontré que ces idées entraînaient respectivement des résultats mortels ou inefficaces.

Il existe cependant une approche mitoyenne entre ces deux extrêmes, où nous devons utiliser les données probantes les plus solides et pertinentes qui sont tirées d'études cliniques aléatoires, quasi expérimentales ou observationnelles pour prendre des décisions éclairées en vue de la mise en place de nouveaux services de pharmacie.

Dans ce numéro du JCPH, deux groupes de pharmaciens décrivent l'approche qu'ils ont utilisée pour mettre sur pied de nouveaux services de pharmacie clinique. Bussières et ses collaborateurs $^{7}$ décrivent la méthode qu'ils ont utilisée pour élaborer un programme de soins pharmaceutiques dans un service d'hémato-oncologie pédiatrique. Mysak et ses collaborateurs $^{8}$ décrivent quant à eux comment ils ont restructuré les services de pharmacie clinique de leur établissement et la portée de cette restructuration sur la satisfaction des parties prenantes. Quelques similitudes importantes entre ces deux projets soulignent le besoin de 
recourir à une approche systématique fondée sur des données probantes dans la mise sur pied d'un nouveau service de pharmacie. Les deux groupes ont amorcé leur projet par une analyse des pratiques actuelles afin de déterminer les lacunes et les besoins importants dans leur établissement respectif. Chacune de ces évaluations était combinée à une revue de la littérature pour retracer les données les plus probantes. Bussières et ses collaborateurs visaient à retracer des études comparatives et d'autres données probantes à l'appui des activités de pharmacie cliniques au sein d'un service d'hématooncologie ${ }^{7}$. Mysak et ses collaborateurs ont quant à eux combiné leur recherche de la littérature à une réunion consensuelle de leurs chefs de file en pratique clinique afin de déterminer un ensemble de services de pharmacie clinique proactifs de base $^{8}$. La deuxième étape importante de la démarche des deux groupes a été de recueillir les commentaires des parties prenantes avant de mettre de l'avant leurs interventions. La dernière étape décrite dans leurs articles a été d'évaluer la portée du nouveau service de pharmacie dans chaque établissement.

Les méthodes utilisées par les deux groupes pour élaborer et évaluer leurs programmes étaient cohérentes avec une méthode d'amélioration de la qualité décrite par diverses organisations $s^{9,10}$. Sommairement, cette méthode comporte six étapes : 1) définir les secteurs prioritaires, 2) déterminer les meilleures pratiques ou un étalon dans la littérature, 3) examiner les habitudes de pratique actuelles pour cerner les lacunes importantes, 4) tenir des discussions avec les parties prenantes pour élaborer une intervention visant à améliorer la pratique actuelle, 5) mettre en œuvre l'intervention et 6) évaluer le programme pour déterminer s'il a entraîné des améliorations au chapitre des résultats cliniques, économiques et humanistes ${ }^{9-11}$. La décision de créer ou de bonifier un service de pharmacie est certes difficile lorsque les ressources en temps, en personnel et en financement sont limitées. Les projets de Bussières et ses collaborateurs ${ }^{7}$ et de Mysak et ses collaborateurs $^{8}$ servent d'exemples sur la manière dont nous pouvons utiliser une approche fondée sur des données probantes pour créer des services de pharmacie clinique. L'emploi judicieux des données les plus probantes et les plus pertinentes pour appuyer ces décisions nous aideront à mettre de l'avant des services de pharmacie qui apporteront des bienfaits importants pour nos patients, nos établissements et notre personnel.

\section{Références}

1. Pratiques organisationnelles requises. Ottawa $(\mathrm{ON})$ : Agrément Canada. Publié au www.accreditation.ca/programmes-d-agrements/qmentum/ pratiques-organisationnelles-requises/. Consulté le 15 janvier 2010.

2. Detsky AS, Naglie IG. A clinician's guide to cost-effectiveness analysis. Ann Intern Med 1990;113(2):147-154.

3. Smith GC, Pell JP. Parachute use to prevent death and major trauma related to gravitational challenge: systematic review of randomised controlled trials. BMJ 2003;327(7429):1459-1461.

4. Echt DS, Liebson PR, Mitchell LB, Peters RW, Obias-Manno D, Barker $\mathrm{AH}$, et al. Mortality and morbidity in patients receiving encainide, flecainide, or placebo. The Cardiac Arrhythmia Suppression Trial. N Engl J Med 1991;324(12):781-788.

5. Yusuf S, Sleight P, Pogue J, Bosch J, Davies R, Dagenais G. Effects of an angiotensin-converting-enzyme inhibitor, ramipril, on cardiovascular events in high-risk patients. The Heart Outcomes Prevention Evaluation Study Investigators. N Engl J Med 2000;342(3):145-153.

6. Bosch J, Yusuf S, Gerstein HC, Pogue J, Sheridan P, Dagenais G, et al. Effect of ramipril on the incidence of diabetes. $N$ Engl $J$ Med 2006;355(15):1551-1562.

7. Bussières JF, Robelet A, Therrien R, Touzin K. Démarche pour la mise à niveau d'un secteur de soins pharmaceutiques : le cas de l'hémato-oncologie pédiatrique. Can J Hosp Pharm 2010;63(2):119-129.

8. Mysak TM, Rodrigue C, Xu J. Care providers' satisfaction with restructured clinical pharmacy services in a tertiary care teaching hospital. Can J Hosp Pharm 2010;63(2):105-112.

9. McQueen L, Mittman BS, Demakis JG. Overview of the Veterans Health Administration (VHA) Quality Enhancement Research Initiative (QUERI). J Am Med Inform Assoc 2004;11(5):339-343.

10. King KM, Bungard TJ, McAlister FA, Ackman ML, Simpson SH, Teo KK, et al. Quality improvement for CQI. Prev Med Manag Care 2000;1(3):129-137.

11. Kozma CM, Reeder CE, Schulz RM. Economic, clinical, and humanistic outcomes: a planning model for pharmacoeconomic research. Clin Ther 1993;15(6):1121-1132.

Scot H Simpson, B.S.P., Pharm. D., M. Sc., est professeur agrégé de la Faculté de pharmacie et des sciences pharmaceutiques de l'Université de I'Alberta, à Edmonton, en Alberta, et reçoit le soutien des Instituts de recherche en santé du Canada à titre de nouveau chercheur. II est également rédacteur adjoint du $\mathrm{JCPH}$.

\section{Address correspondence to:}

\section{D' Scot H Simpson}

Faculty of Pharmacy and Pharmaceutical Sciences

2125A Dentistry/Pharmacy Centre

University of Alberta

Edmonton (AB) T6G 2N8

Courriel : ssimpson@pharmacy.ualberta.ca 